\section{Addition of Simvastatin to Carvedilol and Endoscopic Variceal Ligation improves rebleeding and survival in patients with Child-Pugh A and B class but not in Child Pugh C class}

\author{
Sanjeev Kumar Jha*, Kuldeep Saharawat, Ravi Keshari, Praveen \\ Jha, Shubham Purkayastha and Ravish Ranjan \\ Department of Gastroenterology, Indira Gandhi Institute of Medical Sciences, Patna, India
}

\section{Summary}

Background: Even with current standard treatment after variceal bleeding which includes combination of nonselective b-blockers and repeated endoscopic variceal ligation, the risk of rebleeding and mortality are high. Statins exhibit an antifibrotic effect and improves HVPG. We evaluated whether addition of simvastatin to carvedilol plus EVL therapy reduces variceal rebleeds or death in patients with cirrhosis.

Method: Patients with a variceal bleed 5 to 10 days before were randomly assigned to groups A [carvedilol $(n=69)$ ] or group B [carvedilol (maximum dose $-12.5 \mathrm{mg})$, and simvastatin $(40 \mathrm{mg} /$ day) $(n=65)]$. Primary end points were variceal rebleeding or death. Secondary end points were new complications of portal hypertension and serious adverse effects of drugs.

Results: During a mean follow-up of $49.05 \pm 25.74$ weeks, composite end point i.e. rebleeding or death developed in 23 patients (33.3\%) in group A and 12 patients $(18.5 \%)$ in group B [HR for simvastatin $=0.512 ; 95 \% \mathrm{Cl}: 0.254-1.030 ; p=0.06$ ]. In subgroup analysis by excluding patients of Child C class, 18 patients $(34.6 \%)$ in group $A$ and 7 patients $(13.6 \%)$ in group $B$ developed composite end point [HR for simvastatin $=0.369 ; 95 \% \mathrm{Cl}: 0.154-0.887 ; p=0.026$ ]. $17.4 \%$ and $15.4 \%$ patients in group $A$ and $B$ developed additional secondary complication $[\mathrm{HR}=0.86 ; 95 \% \mathrm{Cl}$ : $0.345-2.161 ; p=0.75)$. No simvastatin induced significant adverse effects were found.

Conclusion: Addition of simvastatin to carvedilol and EVL may reduce the rebleeding and death in patients with less advance liver disease.

\section{More Information}

\begin{abstract}
*Address for Correspondence: Sanjeev Kumar Jha, Associate Professor, Department of Gastroenterology, Indira Gandhi Institute of Medical Sciences, Patna, India, Tel: +91-6122297631; Email: drsanjeevjha2010@gmail.com

Submitted: 05 August 2019

Approved: 19 September 2019

Published: 20 September 2019

How to cite this article: Jha SK, Saharawat K, Keshari R, Jha P, Purkayastha S, et al. Addition of Simvastatin to Carvedilol and Endoscopic Variceal Ligation improves rebleeding and survival in patients with Child-Pugh A and B class but not in Child Pugh C class. Ann Clin Gastroenterol Hepatol. 2019; 3: 020-026.
\end{abstract}

DOI: dx.doi.org/10.29328/journal.acgh.1001010

Copyright: @ 2019 Jha SK, et al. This is an open access article distributed under the Creative Commons Attribution License, which permits unrestricted use, distribution, and reproduction in any medium, provided the original work is properly cited

Check for updates

\section{Introduction}

Portal hypertension is the hemodynamic abnormality associated with the most severe complications of cirrhosis, including ascites, hepatic encephalopathy and variceal bleeding. Variceal bleeding is a medical emergency associated with a mortality that, in spite of recent progress, is still in the order of $10 \%-20 \%$ at 6 weeks [1]. Patients with portal hypertension, who have bled from esophageal varices, have a $60 \%-80 \%$ risk of variceal rebleeding at 1 year, if no therapy for prevention of variceal rebleeding is instituted [2]. The current first-line standard treatment after variceal bleeding is the combination of nonselective b-blockers (NSBBs) and repeated endoscopic variceal ligation (EVL) until variceal eradication. With this treatment, the 2-year risk of rebleeding (30\%) and mortality (25\%) are still high [3]. The hepatic venous pressure gradient (HVPG) is significantly associated with the risk of variceal bleeding and liver decompensation. A reduction in HVPG by $20 \%$ of baseline results in a significant reduction in the bleeding risk and a reduction to less than $12 \mathrm{~mm} \mathrm{Hg}$ virtually abolishes the risk [4]. Propranolol, a NSBB, significantly reduces HVPG and is currently recommended for the prevention of primary and secondary variceal bleeding. However, it achieves the target HVPG reduction only in approximately one-third of patients, possibly because the increase in porto-collateral resistance impairs the reduction in portal pressure $[5,6]$. 
Carvedilol is a potent NSBB with an anti-alfa-1 adrenergic activity, which confers to the drug a potential for a higher portal pressure reduction compared with other NSBB [7]. A systematic review of randomized controlled trials (RCTs) showed that carvedilol reduces HVPG significantly more than propranolol. Mean difference between the percentage of HVPG reduction achieved with propranolol and carvedilol is -7.24 (CI -10.50 to -3.97 ) in favour of carvedilol [7]. A recent RCT showed that carvedilol is as effective as nadolol plus isosorbide mononitrate for secondary prevention of variceal bleeding support a potential for a clinical benefit from carvedilol [8]. In addition, carvedilol may achieve a hemodynamic response in $56 \%$ of patients failing to respond to propranolol [9]. Hence Carvedilol may replace propranolol as a NSBB in secondary prophylaxis of variceal bleeding.

Unfortunately, many patients do not respond to such therapy or are intolerant to them. Therefore other drug required in clinical practice that can slow progression of fibrosis and improve portal hemodynamics.

Statins are 3-hydroxy-3-methyl-glutaryl-coenzyme A (HMGCoA) reductase inhibitor usually prescribed as a lipid lowering agents. However, there is accumulating evidence that statins have multiple secondary effects both related and unrelated to their lipid-lowering effect. In patients with chronic liver disease, statins exhibit an antifibrotic effect possibly through the prevention of hepatic sinusoidal micro thrombosis [10]. They show an anti-inflammatory effect in the inflammatory response caused by endotoxins, angiotensin II or hypovolemia [11]. A multicentre placebo controlled RCT showed statins induced improvement in HVPG [12].

Furthermore, statins were identified by the Baveno VI consensus workshop as promising new medications in the management of portal hypertension [13].

We conducted a RCT of combination of carvedilol plus simvastatin versus carvedilol alone in the secondary prophylaxis of variceal rebleeding from esophageal varices in patients with liver cirrhosis. The main objectives were to evaluate, whether carvedilol plus simvastatin therapy reduces the incidence of variceal rebleeds and improves survival compared with carvedilol alone in patients with liver cirrhosis who had bled from esophageal varices in the past. The adverse effects of the drug therapy were also carefully monitored.

\section{Patients and Methods}

This was a single-centre, prospective, open label and randomized controlled study conducted in the gastroenterology department of the Indira Gandhi institute of medical sciences, Patna, India. The study was approved by the institutional ethics committee. All cirrhotic patients with history of hematemesis and/or melena in between 5 and 10 day and proven to have esophageal varices as the bleeding source were included in this study. Cirrhosis was diagnosed on the basis of clinical, radiological, or laboratory parameters and/or liver biopsy.

Following patients were excluded:

1. Age $<18$ years or $>75$ years

2. Pregnant or lactating women

3. Non cirrhotic cause of variceal bleeding

4. History ofprevious shuntsurgery orTIPS(Transjugular intrahepatic portosystemic shunt) procedure

5. Hepatocellular carcinoma

6. Creatinine $>2 \mathrm{mg} / \mathrm{dL}$

7. Contraindication for statins

8. HIV infection

9. Bleeding due to gastric varices

10. Complete portal vein thrombosis

11. Patients already on NSBB or statins

12. History of myopathy

13. Systolic blood pressure (SBP) $<90 \mathrm{~mm} \mathrm{Hg}$ or Pulse rate $<55$ per minute.

\section{Presence of severe systemic illness}

The study was conducted between January 2017 and August 2018. All patients underwent endoscopic variceal ligation (EVL) at the first endoscopy session. Ligation was done using a multiband ligator. In each session, as many bands as possible were placed on the varices in the lower $5-7 \mathrm{~cm}$ of the esophagus, the number varying from 2 to 6 . EVL was done at intervals of 3-4 weeks until the varices were obliterated. Once varices were eradicated, repeat endoscopy was done at 3-month intervals to check for recurrence of varices. Patients were randomized using computer generated random numbers into carvedilol (Group A) or carvedilol plus simvastatin (Group B). All patients were started Carvedilol at a dose of $6.25 \mathrm{mg} /$ day. Dose of carvedilol was increased to $12.5 \mathrm{mg}$ /day over 7 days if patients tolerated the dose. The dose was reduced if any of the following occurred: SBP $<90 \mathrm{~mm} \mathrm{Hg}$, heart rate $<55$ beat per minute or other serious adverse effects. Group B patients were started simvastatin at $20 \mathrm{mg}$ and titrated to $40 \mathrm{mg}$ after 7 day if initial dose was tolerated. Patients were followed at one month and every 3 months thereafter or when developed any symptoms. Drug compliance was ascertained by interviewing the patient and by checking empty drug rapper. The study medication was stopped if the patients develop the primary end point or toxicity associated with the medication. An asymptomatic increase in alanine aminotransferase (ALT), aspartate 
aminotransferase (AST), or creatine kinase $>3$-fold baseline in absence of other reasonable explanation were considered as significant simvastatin related drug toxicity. All patients with variceal rebleeding were started on terlipressin immediately. After hemodynamic stabilization, endoscopy and if needed, EVL was performed to stop the bleeding. The vasoactive drugs were continued for 5 days.

\section{Treatment end points}

The primary end point was a composite end point defined as variceal rebleeding or death. Two major secondary end points were death and variceal rebleeding. Other secondary end points were development of complication related to underlying liver disease and development of serious adverse effects that required the discontinuation of therapy.

\section{Sample size calculation and statistical analysis}

This study was designed to compare the risks of variceal rebleed or death as calculated by Kaplan-Meier curves for carvedilol versus carvedolol plus simstatin therapy. The probability of rebleed or death on carvedilol therapy was assumed to be $36 \%$ at 1 year. It was hypothesized that carvedilol plus simstatin therapy would reduce the risk to $20 \%$. Using a 1-tailed test with $\alpha$ value of .05 and power (1_ $\beta$ ) of 0.80 , the required sample size would be 162 , i.e., 81 in each group. Quantitative data were expressed as mean $( \pm \mathrm{SD})$ or median (range) and analyzed using Independent Sample $\mathrm{T}$ test. Qualitative data were expressed in frequency and analyzed by Kruskal Wallis test. The primary outcome was analyzed as a time to event variable, the survival function was estimated by means of the Kaplan-Meier method and groups were compared with the Stratified Log-Rank test and hazard ratios (HRs) (95\% confidence interval [CI]) were calculated using the Cox Regression model. Statistical significance was established at a $p<0.05$. Subgroup analysis was done for patients with CTP score $\leq 9$ or $>9$ separately. Statistical analysis was done using the SPSS 17.0 statistical package (IBM).

\section{Results}

As outlined in figure 1, this study enrolled 164 patients which were randomized into carvedilol (Group A, $\mathrm{n}=82$ patients) and carvedilol plus simvastatin (Group B, $\mathrm{n}=$ 82 patients). 13 patients in group $A$ and 17 patients in group B were excluded due to the causes mentioned in figure 1. Therefore, 69 patients in group A and 65 patients in group B entered into final analysis. Mean age of patient was $45.46 \pm 14.74$ year and $71.6 \%$ were male. Common etiologies of cirrhosis were alcohol related, hepatitis B related and cryptogenic seen in $31.3 \%, 31.3 \%$ and $24 \%$ cases respectively. Mean Child-Turcotte-Pugh (CTP) score was 8.4 \pm 1.4 and $22.4 \%$ patients belonged to Child C class. Mean model for end-stage liver disease (MELD)-Na score was 15.8 \pm 6.1 . The baseline characteristics of patients were similar in the 2 groups (Table 1). The mean follow-up was $48.21 \pm$ 27.92 weeks in group A and $49.95 \pm 23.38$ weeks in group B $(p=0.7)$.

\section{Treatment end points}

During mean follow-up of $49.05 \pm 25.74$ weeks, composite end point i.e. rebleeding or death developed in 23 patients $(33.3 \%)$ in group A and 12 patients $(18.5 \%)$ in group B [Hazard ratio (HR) for simvastatin $=0.512 ; 95 \%$ confidence interval (CI): $0.254-1.030 ; p=0.06$ ]. The actuarial probability of developing composite end point at 48 week was $31 \%$ in group A and 19\% in group B (Log rank test $=0.053$ ) (Figure 2). 27 patients $(20.14 \%)$ developed rebleeding. 18 of 69 (26.1\%) in group A and 9 of 65 (13.8\%) in group B developed rebleeding [HR for simvastatin $=0.474 ; 95 \% \mathrm{CI}$ ): $0.213-$ $1.057 ; p=0.07] .24$ patients (17.9\%) died during this period.

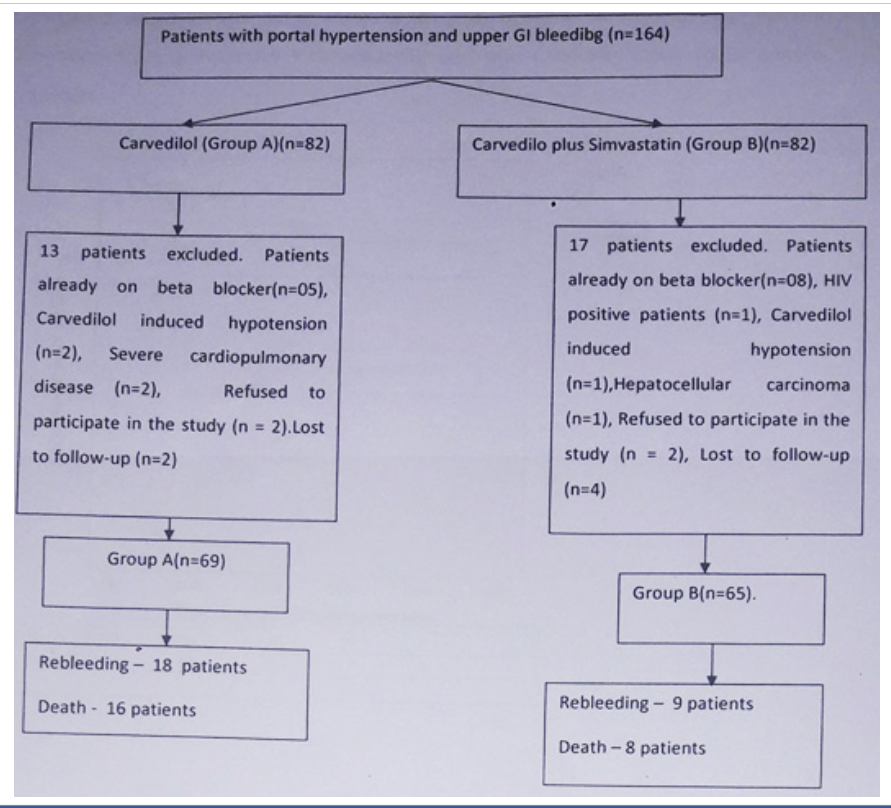

Figure 1: Flow chart of the patients entered into study for analysis.

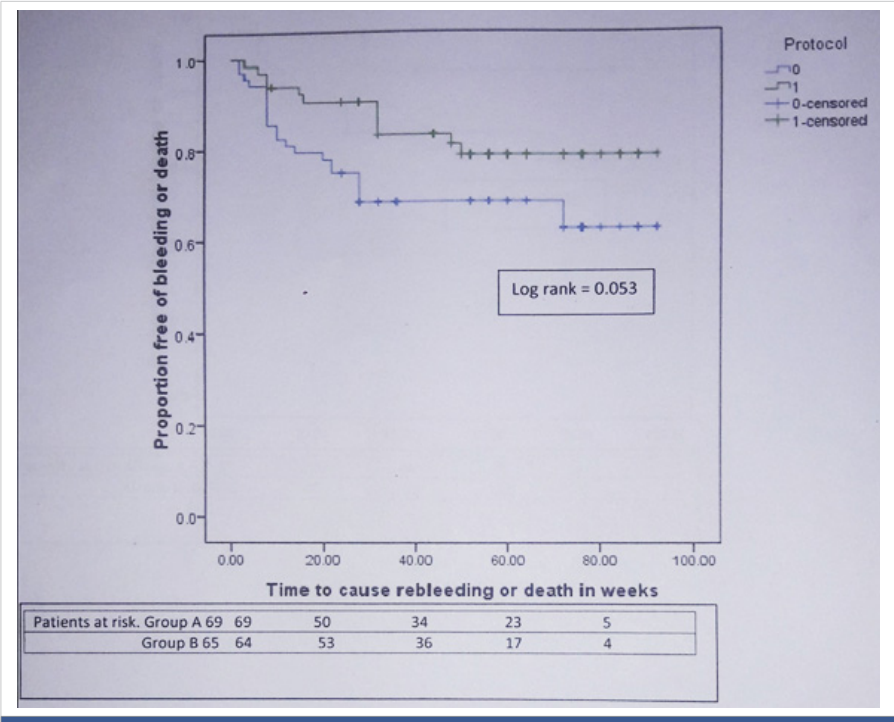

Figure 2: Kaplan-Meier curve showing the probability of developing rebleeding or death in patients from simvastatin + carvedilol (green) and carvedilol alone (blue) groups. 


\begin{tabular}{|c|c|c|c|}
\hline Baseline characteristics & Carvedilol $(n=69)$ & Carvedilol plus Simvastatin( $(=65)$ & $P$ value \\
\hline Age in year (Mean $\pm S D$ ) & $46.02 \pm 14.18$ & $44.86 \pm 15.40$ & 0.65 \\
\hline Sex (Male: Female) & 51:18(73.9\%: $26.1 \%)$ & $45: 20(69.2 \%: 30.8 \%)$ & 0.55 \\
\hline Etiology & & & 0.17 \\
\hline Alcohol (n) & 25 & 17 & \\
\hline Hepatitis B (n) & 23 & 19 & \\
\hline Hepatitis C (n) & 3 & 6 & \\
\hline Cryptogenic (n) & 14 & 18 & \\
\hline Other (n) & 4 & 5 & \\
\hline MELD-Na Score (Mean \pm SD) & $16.49 \pm 5.75$ & $15.03 \pm 6.46$ & 0.17 \\
\hline CTP Score (Means) & $8.52 \pm 1.54$ & $8.29 \pm 1.29$ & 0.35 \\
\hline CTP (A:B:C)\% & 13:62.4:24.6 & $9.2: 70.8: 20$ & \\
\hline Presence of Ascites & $81.2 \%$ & $81.5 \%$ & 0.95 \\
\hline Presence of Hepatic encephalopathy & $5.8 \%$ & $7.7 \%$ & 0.66 \\
\hline MAP in $\mathrm{mmHg}($ Mean $\pm \mathrm{SD})$ & $85.48 \pm 6.23$ & $84.40 \pm 5.83$ & 0.80 \\
\hline Median grade of esophageal varices (Range) & $3(2-4)$ & $3(2-4)$ & 0.36 \\
\hline Presence of Gastric varices & $31.9 \%$ & $24.6 \%$ & 0.35 \\
\hline Presence of severe PHG & $23.2 \%$ & $21.5 \%$ & 0.45 \\
\hline S. Bilirubin in $\mathrm{mg} / \mathrm{dl}($ Mean $\pm \mathrm{SD})$ & $1.77 \pm 1.58$ & $1.89 \pm 1.56$ & 0.64 \\
\hline Albumin in gm/dl (Mean $\pm \mathrm{SD})$ & $2.66 \pm 0.41$ & $2.78 \pm 0.49$ & 0.13 \\
\hline INR (Mean \pm SD) & $1.52 \pm 0.38$ & $1.44 \pm 0.44$ & 0.26 \\
\hline ALT in IU/L (Mean $\pm S D)$ & $54 \pm 57$ & $55 \pm 48$ & 0.90 \\
\hline Hemoglobin in g/dl (Mean \pm SD) & $7.76 \pm 2.36$ & $7.43 \pm 1.49$ & 0.35 \\
\hline Platelet $(\times 109$ cells $/ L)($ Mean \pm SD) & $92 \pm 45$ & $84 \pm 39$ & 0.27 \\
\hline Leukocytes $(\times 109$ cells/L) $($ Mean \pm SD) & $5624 \pm 3010$ & $4915 \pm 2907$ & 0.17 \\
\hline Creatinine in mg/dl $($ Mean $\pm S D)$ & $1.09 \pm 0.36$ & $1.01 \pm 0.31$ & 0.20 \\
\hline Sodium in $\mathrm{meq} / \mathrm{L}($ Mean $\pm \mathrm{SD})$ & $133 \pm 4$ & $133 \pm 6$ & 0.85 \\
\hline Carvedilol dose in mg & $10.01 \pm 2.49$ & $10.77 \pm 2.41$ & 0.07 \\
\hline Follow-up period in weeks & $48.21 \pm 27.92$ & $49.95 \pm 23.38$ & 0.70 \\
\hline
\end{tabular}

Abbreviations: SD-Standard Deviation, n-Number, mg-milligram, CTP-Child Turcotte Pugh, MELD-Na-Model for end-stage liver disease-sodium, MAP- Mean Arterial Pressure, ALT-Alanine Amino Transferase, PHG-Portal Hypertensive Gastropathy, INR-International Nationalized Ratio.

16 of $69(23.18 \%)$ in group A and 8 of 65 (12.3\%) in group B died [HR for simvastatin $=0.547 ; 95 \%$ CI: $0.234-1.281 ; p$ $=0.16]$. In group A, 6 patients died from variceal rebleeding whereas one patient died from variceal rebleeding in group B. Other causes of death were progression of liver disease (group A-7, group B-3 patients), hepatorenal syndrome (group A-4, group B-2 patients), hepatic encephalopathy (group A-2, group B-1 patient) and acute on chronic liver failure (group B-2 patients). The actuarial probability of rebleeding at 48 week was $27 \%$ in group $A$ and $15 \%$ in group B (Log rank test $=0.06)$ (Figure 3). Similarly the actuarial probability of death at 48 week was $20 \%$ in group A and $11 \%$ in group B ( Log rank test $=0.15)$ (Figure 4$)$.

We have done a subgroup analysis by excluding patients of Child C class. 52 patients in each group had CTP score 9 or less. 18 of $52(34.6 \%)$ in group A and 7 of $52(13.6 \%)$ in group B developed composite end point [HR for simvastatin $=0.369 ; 95 \%$ CI: $0.154-0.887 ; p=0.026]$. The actuarial probability of developing composite end point at 48 week was $31 \%$ in group A and 15\% in group B (Log rank test = 0.018) (Figure 5). 14 of 52 (26.9\%) in group $A$ and 5 of 52 $(9.6 \%)$ in group B developed rebleeding [HR for simvastatin $=0.323 ; 95 \%$ CI: $0.116-0.897 ; p=0.03] .11$ of $52(21.2 \%)$ in group A and 3 of 52 (5.8\%) in group B died during this period [HR for simvastatin $=0.287 ; 95 \%$ CI: $0.080-1.033 ; p=0.05$ ]. Therefore treatment with simvastatin plus carvedilol was associated with $68 \%$ reduction in relative risk of rebleeding and $72 \%$ reduction in relative risk of death as compared to carvedilol alone therapy in patients with Child Pugh A and B class. The actuarial probability of rebleeding at 48 week was $27 \%$ in group A and $12 \%$ in group B (Log rank test $=0.021$ ). Similarly the actuarial probability of death at 48 week was $19 \%$ in group A and 6\% in group B (Log rank test $=0.04$ ).

\section{Additional secondary complication}

$17.4 \%$ and $15.4 \%$ patients in group A and B developed additional secondary complication $[\mathrm{HR}=0.86 ; 95 \% \mathrm{CI}$ : $0.345-2.161 ; p=0.75) .4$ patients in group $A$ and 5 patients in group $B$ developed new onset ascites. 3 and 1 patients in group A and B developed spontaneous bacterial peritonitis. Recurrent HE developed in 2 and 1 patients in group $A$ and $B$ respectively. One patient in group A developed bleeding from antral ulcer. One patient in group B developed portal vein thrombosis. One patient in group B developed hepatocellular carcinoma. HRS developed in 2 patients in group A and 1 patient in group B.

Adverse effect: 3 patients developed carvedilol induced hypotension and were excluded from final analysis. We have not found simvastatin induced significant adverse effects that require drug discontinuation. No significant elevation of CPK level seen in group B. 


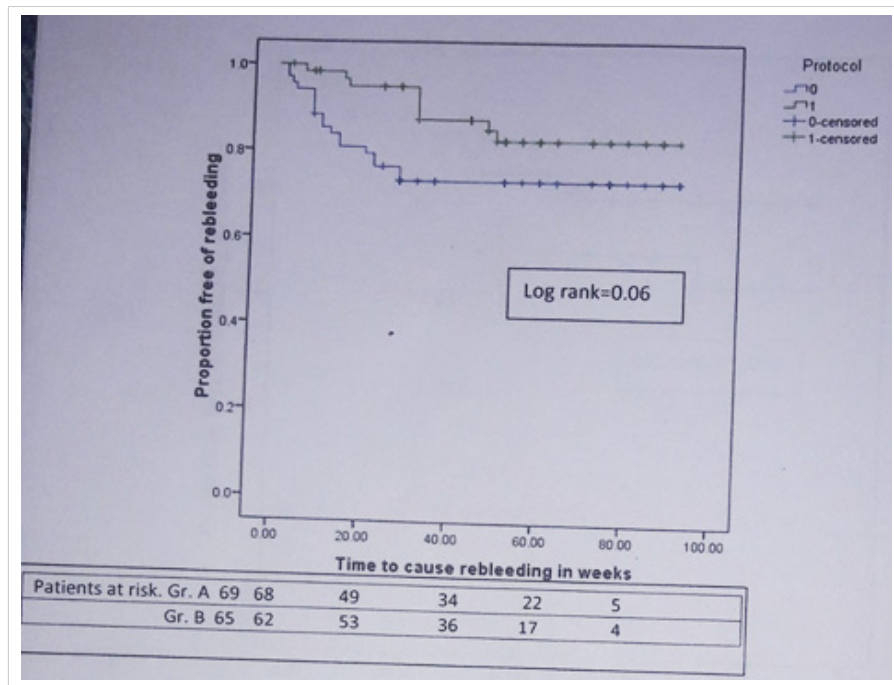

Figure 3: Kaplan-Meier curve showing the probability of developing rebleeding in patients from simvastatin + carvedilol (green) and carvedilol alone (blue) groups $(n=134)$.

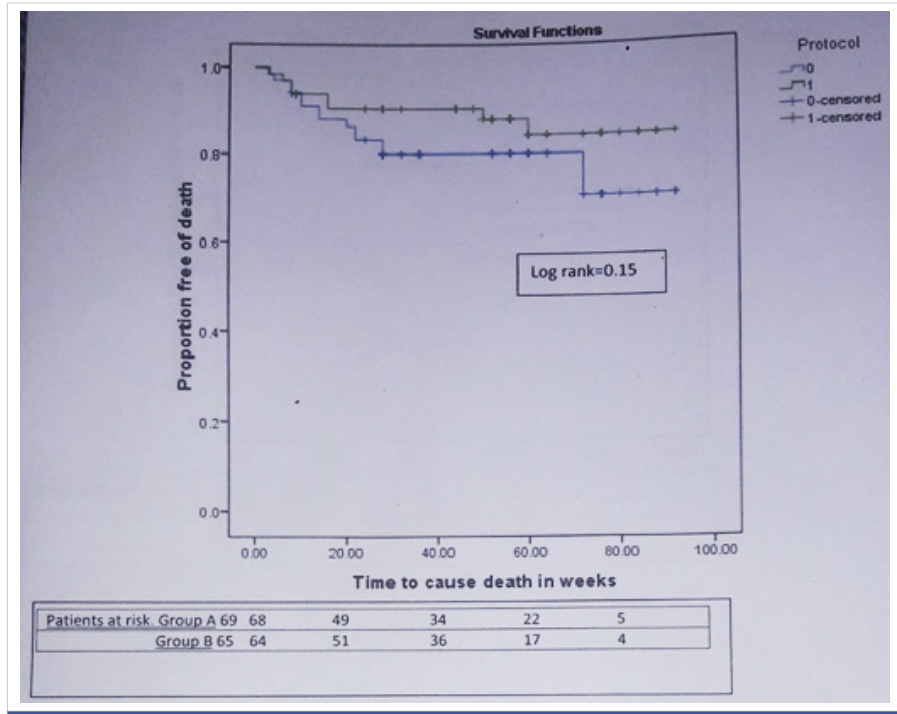

Figure 4: Kaplan-Meier curve showing the probability of developing death in patients from simvastatin + carvedilol (green) and carvedilol alone (blue) groups $(\mathrm{n}=134)$.

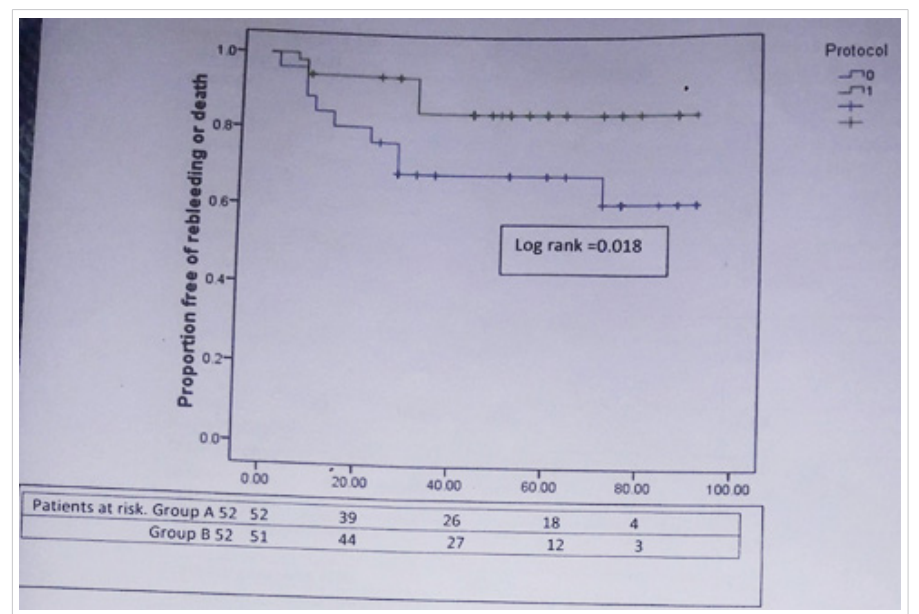

Figure 5: Kaplan-Meier curve showing the probability of developing rebleeding or death in patients from simvastatin + carvedilol (green) and carvedilol alone (blue) groups in patients with Child class $A$ and $B$.

\section{Discussion}

This open label randomized controlled trial showed addition of simvastatin to carvedilol and EVL decreases the rate of rebleeding and mortality after variceal bleeding though the difference was not statistically significant. In patients with Child class A and B, we found that the rebleeding rate and mortality rate are significantly lower in group B as compared to group A. Reduced rate of rebleeding and death especially in Child A and B class may be explained by further decrease in level of HVPG by addition of simvastatin to carvedilol. Besides, antifibrotic and anti-inflammatory activity of statin improves the liver function in patients with chronic liver disease. It also improves the endothelial dysfunction. A RCT showed that Simvastatin significantly decreases HVPG (-8.3\%) without deleterious effects in systemic hemodynamics. Statin treatment led to the decrease of HVPG both in patients treated $(-11 \%, p=0.033)$ and not treated $(-5.9 \%, p=0.013)$ with beta-blocker [12]. Statin treatment did not affect systemic circulation and the incidence of adverse effects was the same in the treatment and control group [12]. Decrease in HVPG by $20 \%$ or more from the baseline was achieved in $55 \%$ of patients taking $40 \mathrm{mg}$ of simvastatin for 3 months [14]. Effects of addition of statin to beta-blocker on clinical outcomes related to portal hypertension have been evaluated in the BLEPS (Bleeding Prevention with Simvastatin) study which was a multicenter double-blind randomized controlled trial. BLEPS study showed less mortality of patients in statin plus propranolol group (9\%) as compared to propranolol group only $(22 \%)(p=0.030)$.The rate of re-bleeding did not differ significantly between the two groups [15].

The potential benefits of statins may extend beyond portal hypertension. Statin modulate the contraction of hepatic stellate cells (HSC) by increasing endothelial nitric oxide synthase (eNOS) and by inducing Kruppel-like factor 2. An over expressed Kruppel-like factor 2 promotes a favourable HSC phenotype and improves liver fibrosis, endothelial dysfunction and portal hypertension $[11,16]$. Simvastatin was able to prevent and lessen the degree of endothelial dysfunction by restoring a more physiologic level of eNOS activity [17]. A human clinical study showed that the simvastatin acutely increases nitric oxide (NO) levels and decreases hepatic resistance [18].

In a matched case control study of 6000 diabetic patients, statin use was associated with a significant reduction in the risk of HCC [19]. Infections among cirrhotic patients have potential to seriously impact life expectancy. Statin use may potentially reduce the risk of infections among patients with cirrhosis [20]. In our study 26 patients in group A and 25 patients in group B has viral etiology. All of them received treatment for their etiologies. Antiviral therapy definitely plays an important role in the progression of liver disease. It is expected that effects of antiviral therapy on progression of liver disease would be comparable in both group considering similar number of patients with viral related liver disease randomized in both group. 
In our study, addition of simvastatin did not show protection against rebleeding and death in Child C patients. $23.5 \%$ of group A and $30.8 \%$ of group B of patients with Child $\mathrm{C}$ class developed rebleeding $(p=0.80)$. Similarly, death seen in $29.4 \%$ of group A and $38.5 \%$ of group B ( $p=0.83$ ) in Child $\mathrm{C}$ patients. Existing data suggest that patients with advance liver disease are too sick to be benefitted from statins and are at higher risk for statin-related complications. A retrospective analysis suggests that the patients with advance cirrhosis may experience more harm than benefit associated with initiating or continuing statin therapy [21]. Abraldes, et al. reported 2 patients on simvastatin developed rhabdomyolysis. Both patients who developed rhabdomyolysis had a bilirubin $>5 \mathrm{mg} / \mathrm{dL}$ [15]. Rhabdomyolysis has been particularly associated with higher doses of simvastatin (80mg) [22]. In our study two patients in simvastatin group had bilirubin $>5 \mathrm{mg} / \mathrm{dL}$. They did not develop rhabdomyolysis. Both of them died due to progression of liver disease. Strength of our study is that it provides valuable data that suggest addition of simvastatin to carvedilol may reduce the incidence of rebleeding and death in Child A and B patients. A limitation of this study is that 30 patients were excluded from the final analysis. As a result, our study might have been underpowered owing to exclusion of significant number of patients. Another limitation of this study is that HVPG measurement was not done and therefore comparative data on reduction of portal pressure is not available. In conclusion, in patients with cirrhosis who recover from an acute variceal bleeding episode, addition of simvastatin to carvedilol and EVL may reduce the rebleeding rate and improves survival in patients with less advance liver disease. Addition of simvastatin is not effective in advance liver disease. Larger study is required to validate our finding.

\section{Acknowledgement}

I would like to thanks Dr. Shishir Kumal (Ph.D), for his help in statistical analysis. He is associate professor of community medicine department in Indira Gandhi institute of medical sciences, Patna.

\section{References}

1. Abraldes JG, Tandon P. Therapies: Drugs, Scopes and Transjugular Intrahepatic Portosystemic Shunt-When and How? Dig Dis. 2015; 33: 524-533.

PubMed: https://www.ncbi.nlm.nih.gov/pubmed/26159269

2. Graham DY, Smith JL. The course of patients after variceal hemorrhage. Gastroenterology. 1981; 80: 800-809.

PubMed: https://www.ncbi.nlm.nih.gov/pubmed/6970703

3. Garcia-Pagan JC, Villanueva C, Albillos A, Bañares R, Morillas R, et al. Nadolol plus isosorbide mononitrate alone or associated with band ligation in the prevention of recurrent bleeding: a multicenter randomized controlled trial. Gut .2009; 58: 1144-1150.

PubMed: https://www.ncbi.nlm.nih.gov/pubmed/19218249

4. Ripoll C, Groszmann R, Garcia-Tsao G, Grace N, Burroughs A, et al. Portal Hypertension Collaborative Group. Hepatic venous pressure gradient predict clinical decompensation in patients with compensated cirrhosis. Gastroenterology. 2007; 133: 481-488. PubMed: https://www.ncbi.nlm.nih.gov/pubmed/17681169

5. Vorobioff J, Picabea E, Villavicencio R, Puccini V, Rossi O, et al. Acute and chronic hemodynamic effects of propranolol in unselected cirrhotic patients. Hepatology. 1987; 7: 648-653.

PubMed: https://www.ncbi.nlm.nih.gov/pubmed/3610045

6. Lebrec D, Hillon P, Munoz C, Goldfarb G, Nouel O, Benhamou JP. The effect of propranolol on portal hypertension in patients with cirrhosis: a hemodynamic study. Hepatology. 1982; 2: 523-527.

PubMed: https://www.ncbi.nlm.nih.gov/pubmed/6981575

7. Sinagra E, Perricone G, Amico MD, Tin F. Systematic review with meta-analysis: the hemodynamic effects of carvedilol compared with propranolol for portal hypertension in cirrhosis. Aliment Pharmacology Therapy. 2014; 39: 557-568.

PubMed: https://www.ncbi.nlm.nih.gov/pubmed/24461301

8. Lo GH, Chen WC, Wang HM, Yu HC. Randomized controlled trial of carvedilol versus nadolol plus isosorbide mononitrate for the prevention of variceal rebleeding. J Gastroenterology Hepatology. 2012; 27: 1681-1687.

PubMed: https://www.ncbi.nlm.nih.gov/pubmed/22849337

9. Thomas Reiberger, Gregor Ulbrich, Arnulf Ferlitsch, Berit Anna Payer, Philipp Schwabl, et al. Carvedilol for primary prophylaxis of variceal bleeding in cirrhotic patients with hemodynamic non-response to propranolol. Gut. 2013; 62: 1634-1641.

PubMed: https://www.ncbi.nlm.nih.gov/pubmed/23250049

10. Martin Janicko, Sylvia Drazilova, Daniel Pella, Jan Fedacko, Peter Jarcuska. Pleiotropic effects of statins in the diseases of the liver. World J Gastroenterol. 2016; 22: 6201-6213.

PubMed: https://www.ncbi.nlm.nih.gov/pubmed/27468210

11. Marrone G, Maeso-Díaz R, García-Cardena G, Abraldes JG, GarcíaPagán JC, et al. KLF2 exerts antifibrotic and vasoprotective effects in cirrhotic rat livers: behind the molecular mechanisms of statins. Gut. 2015; 64: 1434-1443.

PubMed: https://www.ncbi.nlm.nih.gov/pubmed/25500203

12. Abraldes JG, Albillos A, Bañares R, Turnes J, González R, et al. Simvastatin lowers portal pressure in patients with cirrhosis and portal hypertension: a randomized controlled trial. Gastroenterology. 2009; 136: 1651-1658.

PubMed: https://www.ncbi.nlm.nih.gov/pubmed/19208350

13. de Franchi R, Baveno VI. Expanding consensus in portal hypertension Report of the Baveno VI Consensus Workshop: Stratifying risk and individualizing care for portal hypertension. Journal of Hepatology. 2015; 63: 743-752.

PubMed: https://www.ncbi.nlm.nih.gov/pubmed/26047908

14. Pollo-Flores $P$, Soldan M, Santos UC, Kunz DG, Mattos DE, et al. Three months of simvastatin therapy vs. placebo for severe portal hypertension in cirrhosis: A randomized controlled trial. Dig Liver Dis. 2015; 47: 957-963.

PubMed: https://www.ncbi.nlm.nih.gov/pubmed/26321186

15. Abraldes JG, Villanueva C, Aracil C, Turnes J, Hernandez-Guerra M, et al. Addition of Simvastatin to Standard Therapy for the Prevention of Variceal Rebleeding Does NotReduce Rebleedingbut Increases Survival in Patients With Cirrhosis. Gastroenterology. 2016; 150: 1160-1170. PubMed: https://www.ncbi.nlm.nih.gov/pubmed/26774179

16. Trebicka J, Hennenberg M, Laleman W, Shelest N, Biecker E, et al. Atorvastatin lowers portal pressure in cirrhotic rats by inhibition of RhoA/Rho-kinase and activation of endothelial nitric oxide synthase. Hepatology. 2007; 46: 242-253.

PubMed: https://www.ncbi.nlm.nih.gov/pubmed/17596891

17. La Mura V, Pasarin M, Meireles CZ, Miquel R, Rodríguez-Vilarrupla A, et al. Effects of simvastatin administration on rodents with lipopolysaccharide- 
inducedlivermicrovasculardysfunction. Hepatology.2013;57:1172-1181. PubMed: https://www.ncbi.nlm.nih.gov/pubmed/23184571

18. Zafra $C$, Abraldes JG, Turnes J, Berzigotti A, Fernández $M$, et al. Simvastatin enhances hepatic nitric oxide production and decreases the hepatic vascular tone in patients with cirrhosis. Gastroenterology. 2004; 126: 749-755.

PubMed: https://www.ncbi.nlm.nih.gov/pubmed/14988829

19. El-SeragHB,JohnsonML,HachemC,MorganaRO.Statinsareassociated with a reduced risk of hepatocellular carcinoma in a large cohort of patients with diabetes. Gastroenterology. 2009; 136: 1601-1608. PubMed: https://www.ncbi.nlm.nih.gov/pubmed/19208359
20. Motzkus-Feagans C, Pakyz AL, Ratliff SM, Bajaj JS, Lapane KL. Statin use and infections in Veterans with cirrhosis. Aliment Pharmacol Ther. 2013; 38: 611-618.

PubMed: https://www.ncbi.nlm.nih.gov/pubmed/23889738

21. Kaplan DE. The Use of Statins in Patients with Cirrhosis. Gastroenterol Hepatol. 2018; 14: 485-487.

22. Armitage J, Bowman L, Wallendszus $K$, Bulbulia R, Rahimi $K$, et al. Intensive lowering of LDL cholesterol with $80 \mathrm{mg}$ versus $20 \mathrm{mg}$ simvastatin daily in 12,064 survivors of myocardial infarction: a double-blind randomised trial. Lancet. 2010; 376: 1658-1669. PubMed: https://www.ncbi.nlm.nih.gov/pubmed/21067805 